\title{
The Lyman Alpha Forest in Hierarchical Cosmologies
}

\author{
M. Machacek \\ G.L. Bryan \\ P. Anninos \\ A. Meiksin \\ M.L. Norman \\ Y. Zhang
}

This paper was prepared for submittal to the

9th Annual October Astrophysics Conference

College Park, $M D$

October 12-14, 1998

July 2, 1999

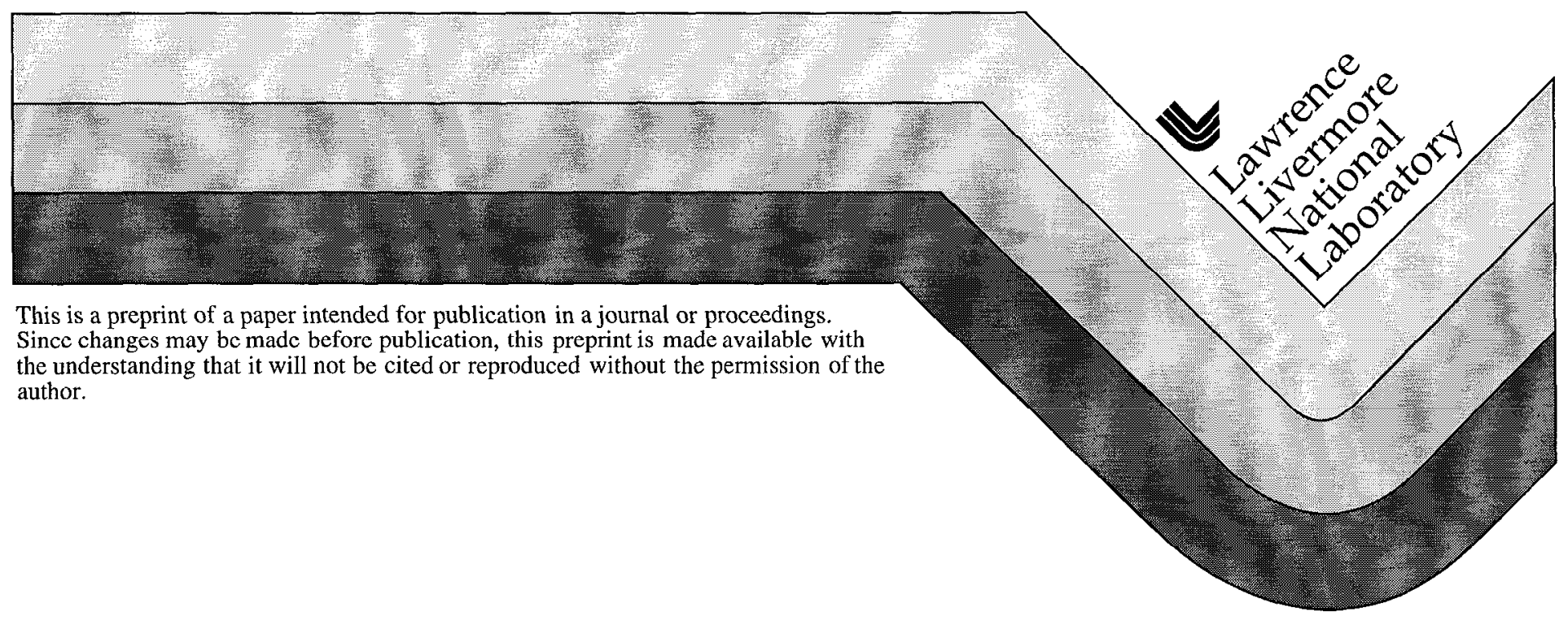




\section{DISCLAIMER}

This document was prepared as an account of work sponsored by an agency of the United States Government. Neither the United States Government nor the University of California nor any of their employees, makes any warranty, express or implied, or assumes any legal liability or responsibility for the accuracy, completeness, or usefulness of any information, apparatus, product, or process disclosed, or represents that its use would not infringe privately owned rights. Reference herein to any specific commercial product, process, or service by trade name, trademark, manufacturer, or otherwise, does not necessarily constitute or imply its endorsement, recommendation, or favoring by the United States Government or the University of California. The views and opinions of authors expressed herein do not necessarily state or reflect those of the United States Government or the University of California, and shall not be used for advertising or product endorsement purposes. 


\title{
The Lyman Alpha Forest in Hierarchical Cosmologies
}

\author{
M. Machacck $^{1}$, G.L. Bryan ${ }^{2}$, P. Anninos ${ }^{3}, \Lambda$. Meiksin $^{4}$, M.L. \\ Norman $^{5}$, and Y. Zhang ${ }^{5}$ \\ ${ }^{1}$ Physics Department, Northeastern University, Boston, MA 02115 \\ ${ }^{2}$ Physics Department, Massachusetts Institute of Technology, Cambridge, MA 02139 \\ 3 \\ University of California, Lawrence Livermore National Laboratory, Livermore, CA 94550 \\ ${ }^{4}$ Institute of Astronomy, University of Edinburgh, Royal Observatory, Blackford Hill, \\ Edinburgh EH9 $3 H J, U K$ \\ ${ }^{5}$ Astronomy Department, University of Illinois at Urbana-Champaign, Urbana, IL 61801
}

\begin{abstract}
The comparison of quasar absorption spectra with numerically simulated spectra from hierarchical cosmological models of structure formation promises to be a valuable tool to discriminate among these models. We present simulation results for the column density, Doppler $b$ parameter, and optical depth probability distributions for five popular cosmological models.
\end{abstract}

\section{INTRODUCTION}

A physical picture of the Ly $\alpha$ forest in hierarchical cosmologies has recently emerged from numerical simulations $[1,2]$ in which the absorbers that give rise to low column density lines $\left(N_{H I}<10^{15} \mathrm{~cm}^{-2}\right.$ at $\left.z \sim 3\right)$ are large, unvirialized objects with sizes of $\sim 100 \mathrm{kpc}$ and densities comparable to the cosmic mean. Since the absorbers grow from the primordial density fluctuations through gravitational amplification, statistics of the forest may be used to test various models of structure formation. We discuss the numerical stability of the statistics against changes in simulation box size and spatial resolution in detail elsewhere. [2] We focus here on examples from our model comparison study [3] in which statistics of the Ly $\alpha$ forest are computed in five cosmological models: the standard cold dark matter model (SCDM), a flat cold dark matter model with nonvanishing cosmological constant (LCDM), a low density cold dark matter model (OCDM), a flat cold dark matter model with a tilted power spectrum (TCDM), and a critical model with both cold dark matter and two massive neutrinos (CHDM). The initial fluctuations, assumed to be Gaussian, 
are normalized using $\sigma_{8 h^{-1}}$ to agree with the observed distribution of clusters of galaxies, although all but SCDM are also consistent with the COBE measurements of the cosmic microwave background. By varying $\sigma_{8 h^{-1}}$ within a given model (SCDM), we also investigate the dependence of the statistics on changes in the fluctuation power spectrum.

The simulation technique uses a particle-mesh algorithm to follow the dark matter and the piecewise parabolic method [4] to simulate gas dynamics. The simulation box length is $9.6 \mathrm{Mpc}$ (comoving) with spatial resolution of $18.75 h^{-1}\left(37.5 h^{-1}\right) \mathrm{kpc}$ for the model (power) comparison studies, respectively. Nonequilibrium effects are followed [5] for six particle species (HI, HII, HeI, HeII, HeIII, and the electron density). We assume a spatially-constant radiation field computed from the observed QSO distribution [6] which reionizes the universe around $z \sim 6$ and peaks at $z \sim 2$. Synthetic spectra are generated along 300 random lines of sight through the volume, including the effects of peculiar velocity and thermal broadening. The spectra are normalized to give a mean optical depth $\langle\tau\rangle=0.3$ at $z=3$ to agree with observation. We do not include the effects of radiative transfer, self-shielding or star formation and so can not address the physics of the highest column density absorbers $\left(N_{H I}>10^{16} \mathrm{~cm}^{-2}\right)$.

\section{FIT DEPENDENT STATISTICS}

The synthetic spectra are fit by Voigt profiles at these low column densities to obtain column densities and Doppler widths for each line. The slope of the column density distribution is insensitive to changes in the size of the simulation volume or grid resolution. [2] Fig. 1 confirms analytic work [7] that the slope of the column density distribution depends primarily on the power in the model at scales $\sim 100-200 \mathrm{kpc}$ and steepens for models with lower power. Each of the five cosmologies in our comparison study agrees with the data at the $3 \sigma$ level, although models (SCDM,LCDM,OCDM) with moderate power at these scales are favored. The Doppler $b$ parameter distributions are determined not only by thermal broadening and peculiar velocities of the absorbers, but also by the Hubble expansion across their width, and require high spatial resolution to be modeled properly. [2,8] The shape of the distribution including the high $b$ tail is well fit by hierarchical models. However, as shown in Fig. 2, observations by Kim, et. al [9] yield median $b$ parameters significantly higher than predicted for the models favored by the column density distributions (LCDM, OCDM, SCDM).

\section{CONCLUSION}

Although hicrarchical cosmologies reproduce the gencral charactcristics of the Ly $\alpha$ absorption spectra quite well, detailed tests of the models may re- 


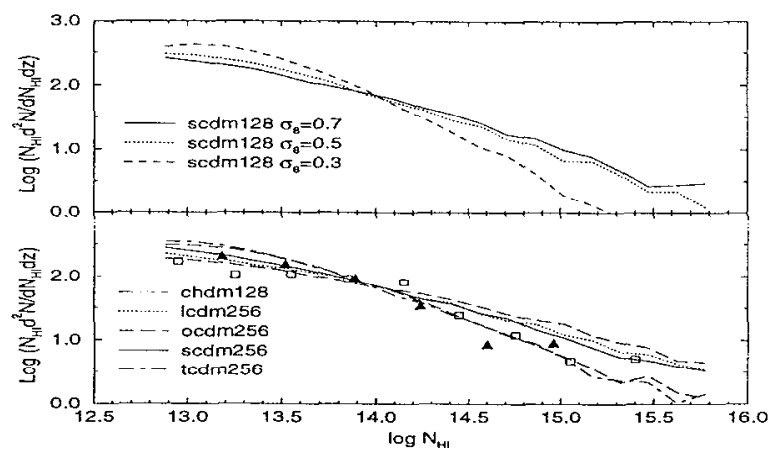

FIGURE 1. HI column density distributions at $z=3$ for lines with $N_{H I}$ in the range $10^{12.8}-10^{16} \mathrm{~cm}^{-2}$. Results for SCDM varying $\sigma_{8 h^{-1}}$ (top); results for the five cosmological models (SCDM, LCDM, OCDM, TCDM, CHDM) are plottcd together with observed data from Kim, et. al [9] (open squares, $z=2.85$ ) and Kirkman \& Tytler [10](filled triangles, $z=2.7)$ (bottom).

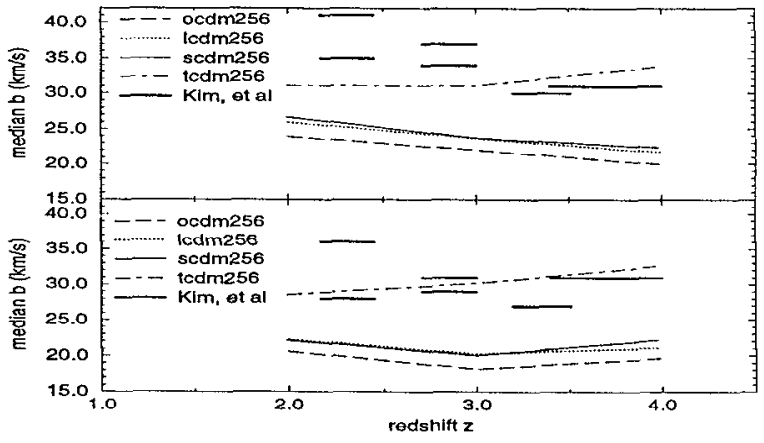

FIGURE 2. 'The evolution of the median of the $b$ parameter distribution with redshift for SCDM, LCDM, OCDM, \& TCDM compared to the observed data by Kim, et. al [9] for lines with $N_{H I}$ in the ranges $10^{13.8}-10^{16} \mathrm{~cm}^{-2}$ (top) and $10^{13.1}-10^{14} \mathrm{~cm}^{-2}$ (bottom). 


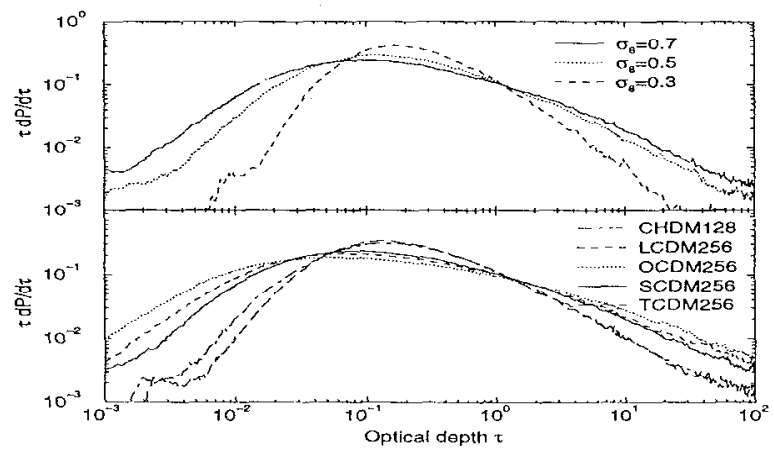

FIGURE 3. Optical depth probability distribution at $z=3$. Results for SCDM with varying power $\left(\sigma_{8 h^{-1}}\right)$ (top); results for SCDM, LCDM, OCDM, TCDM, \& CHDM (bottom).

quire new methods of analysis for the simulations and observations. Statistics derived directly from the flux or optical depth distributions without recourse to any line fitting algorithm are particularly interesting. [11] For example, the optical depth probability distribution, like the column density distribution, is stable to changes in simulation spatial resolution. Fig. 3 shows that the distribution narrows for models with lower power and that the shape of the distribution varies significantly for the different models over the range $0.05<\tau<4$ accessible to observations. Comparison of high quality observations with high resolution simulations using an ensemble of such statistics may soon clarify the physical properties of the intergalactic medium at intermediate redshifts when galaxies wcrc young.

This work is done under the auspices of the Grand Challenge Cosmology Consortium and supported in part by NSF grant ASC-9318185 and NASA Astrophysics Theory Program grant NAG5-3923.

\section{REFERENCES}

1. Cen, R., Miralda-Escude, J., Ostriker, J. P., \& Rauch, M. 1994, ApJ, 437, L9; Zhang, Y., Anninos, P., Norman, M. L., \& Meiksin, A. 1997, ApJ, 485, 496; Hernquist, L., Katz, N., Weinberg, D., \& Miralda-Escude, J. 1996, ApJ, 457, L51

2. Bryan, G. L., Machacek, M., Anninos, P. \& Norman, M. L. 1998, ApJ, in press (astro-ph/9805340)

3. Machacek, M., Bryan, G. L., Meiksin, A., Anninos, P., Thayer, D., Norman, M. L., \& Zhang, Y. 1998 (in preparation)

4. Bryan, G. L., Norman, M. L., Stone, J. M., Cen, R., \& Ostriker, J. P. 1995, Comput. Phys. Comm.,89, 149

5. Anninos, P., Zhang, Y., Abel, T., \& Norman, M. L. 1997, New Astronomy, 2, 209 
6. Haardt, F. \& Madau, P. 1996, ApJ, 461, 20

7. Gnedin, N. Y. 1998, MNRAS, submitted (astro-ph/9706286)

8. Theuns, T. Leonard, A., \& Efstathiou, G. 1998 MNRAS, 297, L49

9. Kim, T.-S., Hu, E. M., Cowie, L. L., \& Songaila, A. 1997, AJ, 114, 1

10. Kirkman, D. \& Tytlcr, D. 1997, $\Lambda$ pJ, 484, 672

11. Rauch, M., Miralda-Escude, J., Sargent, W. L. W., Barlow, T. A., Weinberg, D.H., Hernquist, L., Katz, N., Cen, R., \& Ostriker, J. P. 1997, ApJ, 489, 7

*This work was performed under the auspices of the U.S. Department of Energy by Lawrence Livermore National Laboratory under contract No. W-7405-Eng-48. 